\title{
Mortalidad por sida en Uruguay: perfil de las personas fallecidas en 2014
}

Susana Cabrera*, Daniel Pérez*, Juan José Merét, Victoria Frantchezł, Carolina Iglesias§, Elisa Cabeza§

\section{Resumen}

Introducción: el diagnóstico precoz del virus de la inmunodeficiencia humana y el acceso a un tratamiento antirretroviral temprano y continuado son estrategias clave para evitar el fallecimiento por sida. En Uruguay, desde el año 2005, luego de un ascenso continuo, se observa una estabilización de la mortalidad por sida, no presentando el descenso previsto. Se realizó un estudio con el objetivo de caracterizar el perfil clínico-epidemiológico de la población fallecida por sida en 2014 en Uruguay y valorar la distribución de las muertes en la cascada de continuo de atención.

Método: retrospectivo y observacional, en base a información de registros en certificados de defunción e historias clínicas de personas de edad igual o mayor a 18 años fallecidas por sida durante el año 2014.

Resultados: de los 175 fallecidos, se accedió a 124 registros, correspondiendo a 105 las muertes por sida; $77 \%$ del subsector público y $68 \%$ varones. Se caracterizó por ser una población de adultos jóvenes ( $43,7 \pm 11,6$ años) y con condiciones de vulnerabilidad (alguna vez consumo de drogas 47,1\%; situación de calle y privación de libertad 15,4\%; 37,5\% beneficiarios de planes sociales). El $71,4 \%$ presentó diagnóstico tardío y el 37,1\% falleció en el primer año del diagnóstico. El $60 \%$ falleció sin haber logrado contacto o continuidad en la atención (11,4\% previnculación, 48,6\% sin retención en cuidados).

Conclusiones: la elevada proporción de pacientes fallecidos sin haber establecido vinculación y seguimiento en el sistema de salud, en una población con rasgos de vulnerabilidad social, evidencia la necesidad de adoptar estrategias integrales probadas para mejorar el acceso y la continuidad de la atención.

Palabras clave: Mortalidad por sida

Síndrome de inmunodeficiencia adquirida

Diagnóstico tardío

Cascada de atención de VIH

Key words: $\quad$ AIDS mortality

Acquired immunodeficiency syndrome

Delayed diagnosis

HIV care cascade

*Área Programática de ITS/VIH-Sida - DIGESA, MS

† Fondo de Población de las Naciones Unidas - UNFPA.

‡ Cátedra de Enfermedades Infecciosas; Facultad de Medicina, Universidad de la República.

$\S$ Doctora en Medicina. Consultora.

Conflicto de intereses: la Dra. Susana Cabrera es responsable del Área Programática ITS-VIH/Sida desde el año 2011. Daniel Pérez es técnico contratado de dicha área desde el año 2017.

Correspondencia: Dra. Susana Cabrera. Correo electrónico: scabrera@msp.gub.uy

Recibido: 28/1/19

Aprobado: $3 / 7 / 19$ 


\section{Introducción}

La mortalidad de los pacientes con virus de la inmunodeficiencia humana (VIH) ha cambiado desde la introducción del tratamiento antirretroviral (TARV), modificando el perfil epidemiológico, cambiando las causas de muerte y transformándola en una enfermedad crónica y controlable. Con tratamiento apropiado, oportuno y continuo, las personas con VIH pueden alcanzar similar expectativa de vida a la de población sin $\mathrm{VIH}^{(1-5)}$. La marcada reducción de la mortalidad por sida en los últimos años, fenómeno que se observa a partir de 1996, responde a la introducción de la estrategia de TARV de alta eficacia (esquema de tratamiento que requiere la asociación de fármacos antirretrovirales) ${ }^{(6,7)}$. A pesar de esto, el diagnóstico tardío (es decir, bajo recuento de CD4 $\leq 200 \mathrm{cel} / \mathrm{ml}$ en el momento del diagnóstico o enfermedades oportunistas al debut o en los siguientes 12 meses), el retraso en el acceso y la mala adherencia al TARV, la no retención en cuidados (no seguimiento continuo), comorbilidades y situaciones de vulnerabilidad social, siguen impactando negativamente en la morbimortalidad relacionada con el $\mathrm{VIH}^{(8-12)}$.

La mayoría de los estudios de mortalidad evalúan sus determinantes a través del análisis de estas variables contribuyentes a la muerte, pero también resulta de interés estudiar la relación existente entre mortalidad y los diferentes pilares de la cascada del continuo de atención. El concepto continuo de atención o de cuidados corresponde a una serie de hitos clave para lograr que, efectivamente, una cohorte de pacientes obtengan los máximos beneficios individuales con impacto en la salud pública. Constituye una mirada longitudinal de la epidemia que se expresa en indicadores calculados sobre el total de pacientes que se estima tienen la infección por VIH en una región determinada: proporción de pacientes con VIH diagnosticados, proporción de pacientes en TARV, proporción de pacientes con carga viral $(\mathrm{CV})$ indetectable. El conocimiento de que la $\mathrm{CV}$ indetectable es el principal factor asociado a la reducción de la morbimortalidad y de la transmisión de VIH sustenta las metas 90-90-90 (90\% de personas con VIH diagnosticadas, $90 \%$ de las diagnosticadas en TARV, $90 \%$ de la tratadas con CV indetectable $)^{(13,14)}$. Se han definido una serie de indicadores intermedios que permiten alcanzar estas metas: diagnóstico temprano, vinculación y retención en cuidados. La cuantificación y el análisis de cada uno de los pilares constituye una herramienta fundamental para valorar la magnitud de la brecha en cada pilar. En la medida en que se reduzcan las brechas de la cascada y que aumente la proporción de individuos con CV indetectable, mayor será el impacto en la reducción de la mortalidad, así como en la incidencia de nuevas infecciones ${ }^{(13,14)}$.
La cascada fue diseñada para seguir el continuo de cuidado de la vida de los individuos, no incluye la muerte dentro de los hitos medidos. En la mayoría de los estudios de cohortes las muertes son tomadas en cuenta si se producen luego que el individuo entró en la cohorte y si se mantiene en la misma, muchas veces luego del inicio del TARV ${ }^{(7,15-17)}$. De esta forma se pierde información valiosa sobre las muertes que se producen al diagnóstico de la infección por VIH previas a la vinculación y en pacientes no retenidos en cuidados. Los resultados de un estudio realizado en Calgary, Canadá( ${ }^{(18)}$, sugieren que el uso de la cascada, sin considerar la muerte, puede no representar completamente la complejidad de la epidemia de VIH a la hora de diseñar políticas sanitarias o medir sus impactos.

En Uruguay, la mortalidad por sida tuvo un aumento sostenido hasta el año 2005. Desde entonces, no ha habido cambios significativos en la tasa de mortalidad y se mantuvo en alrededor de 5/100.000 habitantes, alcanzando 8/100.000 habitantes en varones y 3/100.000 habitantes en mujeres en $2014^{(19)}$. El Estudio Nacional de Carga Global de Enfermedad en nuestro país ubica al VIH/Sida en el grupo etario de 20 a 64 años, en el décimo lugar como causa de muerte prematura (AVPP), en el quinto lugar en carga de morbilidad (AVAD) y en el octavo lugar en años de vida ajustados por discapacidad (AVISA) $^{(20)}$.

A diciembre de 2014, año al que refiere la presente investigación, se estimaba que el número de personas con VIH (diagnosticadas y no diagnosticadas) ascendía a 12.000. El número de personas diagnosticadas era aproximadamente 9.000 (75\%); bajo TARV, en ese momento, se encontraban 5.300 pacientes (cobertura 44\%), y con CV indetectable 4.200 (35\% del total de infectados $)^{(19)}$. No se cuenta con datos de vinculación y retención en cuidados, ni con estudios nacionales de mortalidad en pacientes con VIH. Estos datos contrastan, por un lado, con la política de acceso universal al TARV que ha tenido el país, y, por otro lado, con los buenos indicadores de salud de Uruguay, y si bien se han realizado en la última década avances sustantivos en políticas, normativas y esfuerzos asistenciales, no se ha logrado impactar en la reducción de la mortalidad por sida.

Durante el año 2015 el Ministerio de Salud definió cinco Objetivos Sanitarios Nacionales 2020, y dentro de estos, la morbimortalidad por VIH/Sida se encontró dentro de los 15 problemas críticos priorizados sobre los cuales concentrar las acciones. En este marco se planificó el presente estudio cuyo objetivo es caracterizar el perfil epidemiológico y clínico de las personas fallecidas por sida en Uruguay durante el año 2014, identificar los principales determinantes asociados a la defunción y 
valorar la distribución de las muertes en cada pilar de la cascada del continuo de cuidados.

\section{Material y método}

Se trata de un estudio retrospectivo, transversal y descriptivo. En la base de datos de mortalidad se identificaron los certificados de muerte del año 2014 de personas mayores de 18 años cuya causa básica de defunción fue sida (códigos B20; B24 de la CIE;10). A partir de los certificados de defunción se identificaron las instituciones donde se produjo el fallecimiento. Por lo tanto, no se relevó información de las instituciones que no respondieron a la invitación, ni tampoco se relevó información de fallecidos cuando estos eran casos únicos en servicios distantes de Montevideo. Se recabaron los datos mediante la revisión de las historias clínicas correspondientes en las instituciones públicas y privadas que aceptaron la invitación a participar del estudio. Cuando el fallecimiento fue en una institución diferente de la institución prestadora del usuario o se produjo en domicilio, se relevaron los datos también de la historia clínica correspondiente a la institución prestadora.

La recopilación de datos se realizó asignando un código a cada fallecido, no registrándose datos filiatorios. Se recabaron variables sociodemográficas (incluyendo características que se asocian a vulnerabilidad), variables relacionadas al diagnóstico, curso de la enfermedad y tratamientos. La base de datos de notificación se utilizó como fuente de información para completar variables al momento del diagnóstico. Las definiciones operativas utilizadas pueden verse en el Anexo.

Se contó con el aval de la Comisión Nacional de Ética en Investigación y el estudio fue declarado de interés por la Dirección General de la Salud (Resolución No 984 de 2016).

Se realizó un análisis univariado determinando la frecuencia absoluta y relativa de todas las variables cualitativas. Para las variables cuantitativas se testeó la normalidad con el test de Schapiro-Willk. Los datos se presentan con la mediana, valores mínimos-máximos y rango intercuartílico. Para testear diferencias de variables cuantitativas entre fallecidos por sida y fallecidos por causas diferentes al sida se utilizaron tests paramétricos y no paramétricos dependiendo de si se rechazaba o no la hipótesis de normalidad.

Se aplicó el test de independencia de chi cuadrado para evaluar posibles relaciones entre variables cualitativas. Se utilizaron tests no paramétricos para comparar la distribución de variables cuantitativas.

Se consideró un nivel de significación de 5\%.

Se realizó un análisis multivariado exploratorio, mediante el ajuste de una regresión logística binaria, para indagar posibles variables predictoras de mortalidad por sida.

Se utilizó el software libre RStudio*.

\section{Resultados}

Cumplieron con los criterios de inclusión 175 fallecidos. Se tuvo acceso a los registros clínicos de 124 de ellos (71\%). Una vez analizados los registros, se determinó que 19 no fallecieron por causas definitorias de sida a pesar de estar categorizados de esta forma en el certificado de defunción. En el Anexo se presentan las definiciones operativas.

En la tabla 1 se describen las características de los 105 fallecidos por sida, de los cuales $67,6 \%$ fueron de sexo masculino, $76,7 \%$ residían en Montevideo y 77,1\% eran usuarios del subsector público. La principal vía de transmisión fue sexual (98\%). En $15(14,3 \%)$ pacientes el fallecimiento se constató en domicilio; $12(14,8 \%)$ del subsector público y $3(12,5 \%)$ del privado. De los 49 (46,7\%) pacientes con antecedentes de consumo de drogas ilícitas, en 12 se registraba el uso de drogas inyectables.

El registro clínico de algunas informaciones relativas a la situación de empleo, escolaridad, situación de pareja fue deficitario.

La mediana de edad al momento del diagnóstico de VIH fue de 35 años y del diagnóstico de sida de 38 años, con una mediana de tiempo entre el diagnóstico y el fallecimiento de cuatro años. En 39 pacientes $(37,1 \%)$ el fallecimiento se produjo en la internación donde se realizó el diagnóstico de VIH o en el primer año posterior al diagnóstico.

Cuando se compararon las características de los fallecidos en domicilio contra los fallecidos en el hospital se encontraron diferencias estadísticamente significativas en tiempo entre el diagnóstico y el fallecimiento: $11,8 \pm 6,5$ años y $5,3 \pm 6,3$ años ( $p<0,0001)$; mal control de VIH $2 / 15$ y $48 / 90$ ( $p=0,004)$; estuvieron en TARV alguna vez $14 / 15$ y $53 / 90(\mathrm{p}=0,001)$, y tuvieron $\mathrm{CV}$ indetectable alguna vez $6 / 15$ y $13 / 83(\mathrm{p}=0,028)$.

Tuvieron un diagnóstico tardío $70(71,4 \%)$ pacientes. Se buscaron variables asociadas al diagnóstico tardío, encontrándose el haber estado en situación de calle (OR 8,55; IC 95\%, 1,14-64,30; p 0,01); tener pareja estable (OR 0,30; IC 95\%, 0, 10-0,90; p 0,03); haber estado privado de libertad (OR 0,39; IC 95\%, 0,16-0,92; p $0,003)$ y el antecedente de uso de drogas (OR 0,64 ; IC $95 \%, 0,44-0,94 ;$ p 0,04$)$.

\footnotetext{
* R Core Team (2016). R: A language and environment for statistical computing. R Foundation for Statitiscal Computing, Vienna, Austria. URL https://www.r-project.org/
} 
Tabla 1. Características sociodemográficas de los fallecidos por sida.

\begin{tabular}{|c|c|c|c|}
\hline Variables ( $n$ con datos) & Unidad de medida & Resultado & \\
\hline Edad al fallecimiento (años) $(n=103)$ & Mediana (Mn-Mx) & $42(21-78)$ & \\
\hline \multirow[t]{2}{*}{ Sexo biológico $(n=105)$} & Masculino & $\mathrm{n}(\%)$ & $71(67,6)$ \\
\hline & Femenino & $\mathrm{n}(\%)$ & $34(32,4)$ \\
\hline \multirow[t]{3}{*}{ Género $(n=105)$} & Masculino & $\mathrm{n}(\%)$ & $70(66,7)$ \\
\hline & Femenino & $\mathrm{n}(\%)$ & $34(32,4)$ \\
\hline & Trans femenina & $\mathrm{n}(\%)$ & $1(0,9)$ \\
\hline \multirow[t]{2}{*}{ Subsector de atención $(\mathrm{n}=105)$} & Público & $\mathrm{n}(\%)$ & $81(77,1)$ \\
\hline & Privado & $\mathrm{n}(\%)$ & $24(22,9)$ \\
\hline \multirow[t]{2}{*}{ Residencia ( $n=103$ ) } & Montevideo & $\mathrm{n}(\%)$ & $79(76,7)$ \\
\hline & Interior & $\mathrm{n}(\%)$ & $24(23,3)$ \\
\hline \multirow[t]{2}{*}{ Lugar de fallecimiento $(n=105)$} & Hospital & $\mathrm{n}(\%)$ & $90(85,7)$ \\
\hline & Domicilio & $\mathrm{n}(\%)$ & $15(14,3)$ \\
\hline Máximo nivel educativo $\leq 9$ años ( $\mathrm{n} 3=9$ ) & $\mathrm{n}(\%)$ & $28(71,8)$ & \\
\hline \multirow[t]{2}{*}{ Trabajo } & Al diagnóstico de $\mathrm{VIH}(\mathrm{n}=68)$ & $\mathrm{n}(\%)$ & $33(48,5)$ \\
\hline & Al fallecimiento $(n=65)$ & $\mathrm{n}(\%)$ & $18(27,7)$ \\
\hline Pareja al fallecimiento $(n=65)$ & $\mathrm{n}(\%)$ & $24(36,9)$ & \\
\hline Beneficiario de planes sociales $(n=80)$ & $\mathrm{n}(\%)$ & $30(37,5)$ & \\
\hline \multirow[t]{2}{*}{ Privación de libertad ( $n=104)$} & Antecedente & $\mathrm{n}(\%)$ & $16(15,4)$ \\
\hline & Al fallecimiento & $\mathrm{n}(\%)$ & $5(4,8)$ \\
\hline \multirow[t]{2}{*}{ Situación de calle ( $n=104$ ) } & Alguna vez en la vida & $\mathrm{n}(\%)$ & $16(15,4)$ \\
\hline & Al fallecimiento & $\mathrm{n}(\%)$ & $5(4,8)$ \\
\hline \multirow[t]{2}{*}{ Consumo de drogas } & Antecedente $(n=104)$ & $\mathrm{n}(\%)$ & $49(47,1)$ \\
\hline & Al fallecimiento $(n=102)$ & $\mathrm{n}(\%)$ & $34(33,3)$ \\
\hline \multirow[t]{7}{*}{ Comorbilidades $(n=102)$} & Diabetes & $\mathrm{n}(\%)$ & $6(5,9)$ \\
\hline & Hipertensión arterial & $\mathrm{n}(\%)$ & $13(12,7)$ \\
\hline & Dislipemia & $\mathrm{n}(\%)$ & $3(2,9)$ \\
\hline & Cardiopatía isquémica & $\mathrm{n}(\%)$ & $1(0,9)$ \\
\hline & Enfermedad renal crónica & $\mathrm{n}(\%)$ & $7(6,9)$ \\
\hline & Enfermedad psiquiátrica & $\mathrm{n}(\%)$ & $15(14,7)$ \\
\hline & Cáncer & $\mathrm{n}(\%)$ & $13(12,7)$ \\
\hline
\end{tabular}

Tuvieron controles habituales de la enfermedad (mensual, cada 2-3 meses y cada 4-6 meses) 52 (49,5\%) pacientes.

Recibieron alguna vez TARV 67 (63,8\%) pacientes y $36(34,3 \%)$ lo recibían al momento del fallecimiento. No hubo diferencias significativas en los pacientes que recibieron TARV alguna vez entre el subsector público y el privado, $63 \%(51 / 81)$ y $67 \%(16 / 24)$, respectivamente. Tampoco se observaron diferencias significativas en los que recibían TARV al momento del fallecimiento, 32,1\% (26/81) en el público y 41,7\% (10/24) en el privado. 
Tabla 2. Características de los pacientes en relación con la infección por VIH.

\begin{tabular}{|c|c|c|c|}
\hline Variables ( $n$ con datos) & Unidad de medida & & Resultado \\
\hline \multirow[t]{3}{*}{ Vía de transmisión ( $n=105)$} & Sexual & $n(\%)$ & $98(93,4)$ \\
\hline & Sanguínea & $n(\%)$ & $6(5,7)$ \\
\hline & Vertical & $n(\%)$ & $1(0,9)$ \\
\hline \multirow[t]{2}{*}{ Coinfecciones ( $n=68)$} & VHB & $n(\%)$ & $1(1,5)$ \\
\hline & VHC & $n(\%)$ & $10(14,7)$ \\
\hline \multirow[t]{2}{*}{ Edad al diagnóstico de VIH (años) ( $\mathrm{n}=101)$} & Mediana (Mn-Mx) & & $35(10-68)$ \\
\hline & Media (DE) & & $37,5(13,5)$ \\
\hline \multirow[t]{2}{*}{ Edad de diagnóstico de sida (años) (n=95) } & Mediana (Mn-Mx) & & $38(10-68)$ \\
\hline & Media (DE) & & $39,9(12,2)$ \\
\hline \multirow[t]{2}{*}{ Edad al fallecimiento (años) (n=103) } & Mediana (Mn-Mx) & & $42(21-78)$ \\
\hline & Media (DE) & & $43,7(11,6)$ \\
\hline \multirow[t]{2}{*}{ Tiempo entre diagnóstico de VIH y fallecimiento (años) ( $n=103)$} & Mediana (Mn-Mx) & & $4(0-24)$ \\
\hline & Media (DE) & & $6,2(6,6)$ \\
\hline \multirow[t]{2}{*}{ Tiempo entre el diagnóstico de VIH y sida (años) ( $n=95)$} & Mediana (Mn-Mx) & & $0(0-19)$ \\
\hline & Media (DE) & & $2,0(5,1)$ \\
\hline \multirow[t]{2}{*}{ Tiempo entre el diagnóstico de sida y fallecimiento (años) $(n=96)$} & Mediana (Mn-Mx) & & $1,0(0-19)$ \\
\hline & Media (DE) & & $3,7(5,1)$ \\
\hline Diagnóstico de VIH durante la internación del fallecimiento $(n=105)$ & $\mathrm{n}(\%)$ & & $30(28,6)$ \\
\hline \multirow[t]{2}{*}{ Tiempo entre el diagnóstico de VIH y el primer control (meses) $(n=74)$} & Mediana (Mn-Mx) & & $2(0-360)$ \\
\hline & Media (DE) & & $14,9(46,7)$ \\
\hline \multirow[t]{6}{*}{ Controles de VIH ( $n=105)$} & Nunca & $n(\%)$ & $43(41,0)$ \\
\hline & Un control & $n(\%)$ & $7(6,7)$ \\
\hline & Mensual & $n(\%)$ & $29(27,6)$ \\
\hline & Cada 2-3 meses & $n(\%)$ & $15(14,3)$ \\
\hline & Cada 4-6 meses & $n(\%)$ & $8(7,6)$ \\
\hline & Anual & $n(\%)$ & $3(2,9)$ \\
\hline \multirow[t]{2}{*}{ Tiempo entre el último control y el fallecimiento (semanas) ( $n=61$ ) } & Mediana (Mn-Mx) & & $12(0-720)$ \\
\hline & Media (DE) & & $61,7(130)$ \\
\hline Pérdidas de seguimiento > 12 meses $(n=70)$ & $\mathrm{n}(\%)$ & & $50(71,4)$ \\
\hline Diagnóstico tardío $(\mathrm{n}=98)$ & $\mathrm{n}(\%)$ & & $70(71,4)$ \\
\hline Primer recuento de $\mathrm{CD} 4<200 / \mathrm{ml}(\mathrm{n}=78)$ & $\mathrm{n}(\%)$ & & $56(71,8)$ \\
\hline Pacientes con enfermedad oportunista al debut del VIH $(n=104)^{1}$ & $\mathrm{n}(\%)$ & & $45(43,3)$ \\
\hline Pacientes con enfermedad oportunista en los primeros 12 meses del diagnóstico $(n=105)$ & $\mathrm{n}(\%)$ & & $10(9,5)$ \\
\hline \multirow[t]{2}{*}{ Primer recuento de $\mathrm{CD} 4 / \mathrm{ml}(\mathrm{n}=78)$} & Mediana (Mn-Mx) & & $88,0(1-1000)$ \\
\hline & Media (DE) & & $181,2(217,6)$ \\
\hline \multirow[t]{2}{*}{ Nadir de $\mathrm{CD} 4 / \mathrm{mm}^{3}(\mathrm{n}=68)$} & Mediana (Mn-Mx) & & $39,5(1-551)$ \\
\hline & Media (DE) & & $73,5(97,8)$ \\
\hline
\end{tabular}


Viene de la página anterior

\begin{tabular}{|c|c|c|c|}
\hline Variables ( $n$ con datos) & Unidad de medida & & Resultado \\
\hline \multirow[t]{2}{*}{ Último recuento de $C D 4 / m l$ previo al fallecimiento $(n=105)$} & Mediana (Mn-Mx) & & $43,0(1-481)$ \\
\hline & Media (DE) & & $84,1(101,9)$ \\
\hline \multirow[t]{2}{*}{ Recibió TARV (n=105) } & Alguna vez & $n(\%)$ & $67(63,8)$ \\
\hline & Al fallecimiento ( $\mathrm{n}$ & $n(\%)$ & $36(34,3)$ \\
\hline \multirow[t]{2}{*}{ Tiempo entre el diagnóstico de VIH y el inicio de TARV (meses) (n=65) } & Mediana (Mn-Mx) & & $14,0(0-240)$ \\
\hline & Media (DE) & & $33,6(44,9)$ \\
\hline \multirow[t]{2}{*}{ Número de planes de TARV ( $n=65)$} & Mediana (Mn-Mx) & & $1,0(1-10)$ \\
\hline & Media (DE) & & $1,5(1,53)$ \\
\hline Buena adherencia en los últimos 12 meses $(n=73)$ & $\mathrm{n}(\%)$ & & $13(17,8)$ \\
\hline \multirow[t]{2}{*}{ Carga viral de VIH suprimida } & Alguna vez $(n=98)$ & $\mathrm{n}(\%)$ & $19(19,4)$ \\
\hline & $\begin{array}{l}\text { Al fallecimiento } \\
(n=105)\end{array}$ & $n(\%)$ & $3(2,8)$ \\
\hline
\end{tabular}

Las causas de fallecimiento con etiología confirmada más frecuentes fueron: micobacterias tuberculosa y no tuberculosa (19), meningoencefalitis por Cryptococcus neoformans (13), neumonía por Pneumocystis jirovecii (10), más de una etiología (9), linfomas (6), toxoplasmosis encefálica (5), bacteriemias por neumococo (2), histoplasmosis (2), leucoencefalopatía multifocal progresiva (1), neumonía por Rhodococcus (1), sepsis por Salmonella (1). El resto de las causas de fallecimiento fueron presuntivamente explicadas por enfermedades oportunistas (EO), predominando la insuficiencia respiratoria, sepsis de origen respiratorio y meningoencefalitis subaguda. "Se desconoce" fue consignado como "enfermedad o estado patológico que produjo la muerte directamente" en siete pacientes, todos en domicilio, con diagnóstico previo de sida.

Las características de los pacientes en relación con la infección VIH se muestran en la tabla 2.

\section{Cascada de atención}

Los fallecimientos en cada uno de los pilares de la cascada se distribuyeron de la siguiente manera: 30 $(28,6 \%)$ al diagnóstico de VIH; $12(11,4 \%)$ previo a la vinculación con el sistema de salud; $51(48,6 \%)$ en pacientes que no lograron retención en cuidados; $9(8,6 \%)$ retenidos recibiendo TARV, y 3 (2,9\%) con CV de VIH suprimida. No se hallaron diferencias estadísticamente significativas entre los fallecidos en cada pilar de la cascada de atención comparados por sexo, procedencia
Montevideo-interior o asistencia subsector público o privado.

\section{Fallecidos por causas no vinculadas a sida}

En 19 pacientes con infección por VIH la causa de muerte no fue un evento definitorio de estadio sida (EO), por lo tanto fueron excluidos del análisis inicial. Las causas identificadas en los registros clínicos fueron: homicidio, suicidio, sepsis respiratoria, hepatopatía crónica, hemorragia digestiva, infección grave por Clostridium dificcile, insuficiencia cardíaca, ataque cerebrovascular isquémico y hemorragia intracerebral, carcinoma anal y broncopulmonar. Por lo tanto, 15,3\% de los certificados codificados sida como causa de muerte, no correspondían a enfermedades incluidas en la definición de sida (CDC 2014)*.

En la tabla 3 se compara la distribución de los fallecimientos en los pilares de la cascada de ambas subpoblaciones.

En la tabla 4 se presenta la comparación de variables cuantitativas dentro de los dos grupos y en la tabla 5 la comparación de las variables categóricas.

El modelo multivariado ajustado encontró variables independientemente asociadas al resultado fallecimiento por sida, con mayor probabilidad en los pacientes no retenidos en cuidados (OR 14,09; IC 95\% 1,946-700,42) y en aque-

* CDC. Revision surveillance case definition for HIV infection - United States 2014. MMWR 2014; 63: 1-10. https://www.cdc.gov/mmwr/preview/mmwrhtml/rr6303a1.htm 
Tabla 3. Distribución del momento de la muerte en cada pilar de la cascada de atención de los fallecidos por sida y los fallecidos por causas no vinculadas a sida.

\begin{tabular}{|c|c|c|c|}
\hline Momento de la muerte & $\begin{array}{c}\text { Fallecidos con eventos definitorios } \\
\text { de sida }(n=105), \%(n)\end{array}$ & $\begin{array}{l}\text { Fallecidos por eventos } \\
\text { no sida }(n=19), \%(n)\end{array}$ & Valor $p$ \\
\hline Al diagnóstico & $28,6(30)$ & $0,2(1)$ & 0,04 \\
\hline Previnculación & $11,4(12)$ & $15,8(3)$ & 0,70 \\
\hline No retención & $48,6(51)$ & $15,8(3)$ & 0,01 \\
\hline Retenido en cuidados & $8,6(9)$ & $21,0(4)$ & 0,11 \\
\hline Con CV suprimida & $2,9(3)$ & $42,1(8)$ & $<, 001$ \\
\hline
\end{tabular}

Tabla 4. Comparación de variables cuantitativas entre fallecidos por sida y fallecidos por causas no vinculadas a sida.

\begin{tabular}{|c|c|c|c|c|c|}
\hline \multirow[t]{2}{*}{ Variables } & \multicolumn{2}{|c|}{$\begin{array}{l}\text { Fallecidos por eventos definitorios } \\
\text { de sida }\end{array}$} & \multicolumn{2}{|c|}{$\begin{array}{l}\text { Fallecidos por eventos } \\
\text { no sida }\end{array}$} & \multirow[t]{2}{*}{$P$} \\
\hline & $N$ & Mediana (RIQ) $(M n-M x)$ & $N$ & Mediana (RIQ) (Mn-Mx) & \\
\hline $\mathrm{CD} 4 / \mathrm{ml}$ al diagnóstico de $\mathrm{VIH}$ & 78 & $88(34-235)(1-1000)$ & 17 & $248(140-550)(73-843)$ & 0,001 \\
\hline Nadir de CD4/ml & 68 & $39(7-105)(1-551)$ & 15 & $150(96-244)(6-450)$ & 0,001 \\
\hline CD4/mlal fallecimiento & 69 & $43(18-106)(1-481)$ & 16 & 359 (185-511) (6-702) & $<, 001$ \\
\hline Edad al diagnóstico de VIH (años) & 101 & $35(27-46)(10-68)$ & 19 & $42(27-49)(20-60)$ & 0,28 \\
\hline Edad al fallecimiento (años) & 103 & $42(35-51)(21-78)$ & 19 & $45(38-61)(27-74)$ & 0,10 \\
\hline Edad al inicio de TARV (años) & 65 & $36(28-43)(11-67)$ & 16 & $42(33-53)(22-62)$ & 0,04 \\
\hline $\begin{array}{l}\text { Tiempo entre el diagnóstico de VIH e inicio de } \\
\text { TARV (meses) }\end{array}$ & 65 & $14(1-54)(0-240)$ & 16 & $19(3-43)(0-120)$ & 0,96 \\
\hline $\begin{array}{l}\text { Tiempo entre diagnóstico de VIH y diagnóstico de } \\
\text { sida (años) }\end{array}$ & 95 & $0(0-3)(0-16)$ & 16 & $0,35(0-3)(0-13)$ & 0,69 \\
\hline Tiempo entre diagnóstico de VIH y muerte (años) & 103 & $4(0-10)(0-24)$ & 19 & $10(2-14)(0-18)$ & 0,12 \\
\hline Tiempo entre diagnóstico de sida y muerte (años) & 96 & $1(0-6)(0-19)$ & 16 & $5(1-13)(0-17)$ & 0,04 \\
\hline $\begin{array}{l}\text { Tiempo entre el diagnóstico de VIH y el primer } \\
\text { control médico (meses) }\end{array}$ & 74 & $2(1-12)(0-360)$ & 14 & $2(1-48)(0-108)$ & 0,78 \\
\hline
\end{tabular}

llos con EO al debut (OR 11,66; IC 95\% 1,35-514,25); y menor probabilidad para el valor de CD4 nadir por cada unidad de aumento (OR 0,989; IC 95\% 0,984-0,999) y $\mathrm{CV}$ indetectable previo al fallecimiento (OR 0,034; IC $95 \% 0,002-0,354)$.

Cuando se evaluó la bondad de ajuste, se encontró que el modelo explica el 55\% de la variabilidad total y logra clasificar correctamente los valores observados en el $91 \%$ de los casos.

\section{Discusión}

El principal hallazgo de nuestro estudio es que aproximadamente la mitad de los fallecimientos por sida $(48,6 \%)$ ocurrieron en personas que no lograron retención en cuidados de salud luego del diagnóstico de VIH, lo que significa que nunca tuvieron un seguimiento clínico continuo. La proporción de pacientes que falleció previo a la vinculación $(11,4 \%)$, sumada a aquellos no retenidos, alcanzó el $60 \%$. 
Tabla 5. Variables categóricas relacionadas al fallecimiento por sida $(n=124)$.

\begin{tabular}{lll}
\hline Variable & OR (IC 95\%) & Valor $p$ \\
\hline Primer recuento de CD4 <100/ml & $1,33(1,11-1,59)$ & 0,003 \\
CD4<100/ml al fallecimiento & $1,68(1,27-2,22)$ & $<, 001$ \\
Sin retención en cuidados al fallecimiento & $1,22(1,06-1,41)$ & 0,01 \\
Mala adherencia al TARV en los últimos 12 & $1,49(1,04-2,14)$ & $<, 001$ \\
meses & & $<, 001$ \\
Carga viral de VIH indetectable alguna vez & $0,65(0,49-0,87)$ & $<, 001$ \\
Carga viral de VIH indetectable al fallecimiento & $0,30(0,11-0,78)$ & 0,04 \\
Diagnóstico de VIH al fallecimiento & $1,2(1,07-1,35)$ & $<, 001$ \\
Enfermedad oportunista al diagnóstico de VIH & $1,24(1,08-1,42)$ & 0,70 \\
Sexo masculino & $1,03(0,86-1,22)$ & 0,08 \\
Atención en subsector público & $1,17(0,95-1,45)$ & 0,36 \\
Residencia en Montevideo & $0,89(0,77-1,03)$ & 0,74 \\
Antecedente de privación de libertad & $1,06(0,88-1,27)$ & 1,00 \\
Antecedente de situación de calle & $0,98(0,79-1,23)$ & 0,13 \\
Antecedente de uso de drogas & $1,14(0,98-1,32)$ & 0,05 \\
$\leq 9$ años de educación formal & $1,39(0,96-2,02)$ & \\
\hline
\end{tabular}

El 29\% de los pacientes falleció en la internación del diagnóstico de VIH, 25 de 30, debido a una EO, lo que destaca el impacto negativo en la sobrevida del diagnóstico tardío de VIH.

La relación temporal entre el diagnóstico de $\mathrm{VIH}$ y la muerte por sida es otro hallazgo importante, ya que la mediana de tiempo fue de cuatro años, en una población donde el $71 \%$ de los pacientes se presentaron con un diagnóstico tardío y $37 \%$ falleció en el primer año del diagnóstico. La proporción de diagnósticos tardíos en fallecidos por sida hallada en nuestro estudio es mayor que la encontrada en la región, reportándose $44 \%$ en un estudio argentino ${ }^{(12)}$.

Encontramos, como variable asociada al diagnóstico tardío, el antecedente de situación de calle, lo que traduce una condición de extrema vulnerabilidad, mientras que tenían menor probabilidad de presentar un diagnóstico tardío las personas con antecedentes de privación de libertad y personas con antecedentes de uso de drogas. Estos hallazgos podrían explicarse por la realización del estudio serológico sistemático de VIH a nivel carcelario desde el año 2005 o la percepción de riesgo elevado, que resulta en una mayor oferta de testeo en los servicios de salud al identificarlos como población clave, en particular en el caso de usuarios de drogas. También se verificó menor probabilidad de diagnóstico tardío en las personas que tenían pareja estable. Si bien una explicación podría ser el factor protector que representa la contención familiar, debe interpretarse este hallazgo con cautela, dado que la información se obtuvo en poco más del $50 \%$ de la población estudiada. No pudo verificarse asociación con otros factores socioculturales, probablemente por el escaso registro de estos en las historias clínicas.

En cuanto al seguimiento y los cuidados, más de dos tercios $(71 \%)$ tuvo al menos una pérdida en el seguimiento. Si bien el $64 \%$ de los pacientes recibió TARV en algún momento de la evolución, solo el 34\% lo recibía al momento del fallecimiento. La CV indetectable, parámetro que mide el éxito terapéutico, fue alcanzada en algún momento en $19 \%$ de los pacientes y solo en el $3 \%$ al momento del fallecimiento. En el $82 \%$ se identificó una mala adherencia al tratamiento en los 12 meses previos al fallecimiento. Estos datos reflejan el impacto negativo que tienen la falta de adherencia y retención en cuidados ${ }^{(9,11)}$.

En cuanto a las características de la población estudiada, la mayoría residía en Montevideo y tres cuartas partes de los pacientes se asistían en el subsector público. La edad mediana al fallecimiento fue de 42 años, en- 
contrándose, por lo tanto, en plena edad productiva. La mayoría de los fallecidos fueron de sexo masculino, en una proporción 2:1 y con una edad promedio al momento del diagnóstico de VIH de 35 años. Si bien la mortalidad por sexo no presentó diferencias estadísticamente significativas, el sexo masculino ha sido un factor de riesgo de mortalidad por sida en varios estudios ${ }^{(12,21)}$. En cuanto al perfil de vulnerabilidad social, encontramos un bajo nivel de educación formal en casi tres cuartas partes de la población con datos en los registros clínicos (72\%); uso de drogas en $47 \% ; 15 \%$ con antecedentes de haber estado o estar privada de libertad; $15 \%$ con el antecedente de haber estado o encontrarse en situación de calle, y $72 \%$ sin trabajo al momento de la internación. Estos datos señalan las condiciones de vulnerabilidad en que se encontraba la mayor parte de la población de fallecidos.

Resulta llamativo que el 14\% de los pacientes haya fallecido en el domicilio, fuera del ámbito hospitalario. Más aún, habiendo presentado mejores resultados en algunas variables clave respecto a los fallecidos en el hospital, por ejemplo, mayor tiempo de sobrevida, haber recibido TARV alguna vez, continuidad de controles, CV indetectable alguna vez en el seguimiento. A lo anterior se agrega que de los 15 pacientes, en siete se consignó como causa final de fallecimiento en el certificado "causa desconocida". Respecto a la cascada del continuo de atención, este grupo de pacientes falleció en el pilar de "no retención", lo que demuestra nuevamente el impacto negativo de la desvinculación del sistema de salud.

La problemática queda aún más al descubierto cuando comparamos las diferencias en la distribución de las muertes en la cascada de las personas que fallecieron por sida y las 19 fallecidas por eventos no relacionados (tabla 3). El 63\% de los fallecidos por eventos no sida presentaron indicadores adecuados de calidad de los cuidados (retención y CV indetectable), mientras que solo el $12 \%$ de los fallecidos por sida alcanzaron estos indicadores. Además, el 42\% alcanzó la indectabilidad viral frente al apenas 3\% ya mencionado en la población fallecida por sida. Si bien inicialmente no se planeó este objetivo, estos hallazgos condujeron a la realización de un análisis multivariado identificando variables independientemente asociadas a la muerte por eventos sida, tanto actuando como factores de riesgo (no retención y EO en el debut) como aquellos que resultan protectores (CD4 nadir y CV indetectable). El número pequeño de pacientes explica los intervalos de confianza amplios, aun así pudieron establecerse asociaciones con suficiente fortaleza estadística.

Los resultados de este estudio muestran la necesidad de implementar estrategias con demostrada eficacia para minimizar las brechas en vinculación-retención, co- mo diagnosticar y tratar (minimizando el tiempo entre diagnóstico y tratamiento), proveer soporte social y psicológico cuando se requiera, utilizar recordatorios de visita (mensajes de texto, llamadas), realizar seguimiento activo (identificar pérdidas de visitas, contacto telefónico previo a las visitas), flexibilización de la agenda de consulta, acompañamiento por pares y la provisión de cuidados en la comunidad $^{(6,22-25)}$.

A las limitaciones propias de los estudios retrospectivos se suman algunas dificultades. Por un lado, encontramos una omisión importante en los registros clínicos de datos vinculados a algunas características sociales (empleo, educación, familia, prestaciones, etcétera), que en general definen situaciones de vulnerabilidad que ponen en juego la adherencia al TARV y a los cuidados. Por otro lado, se destaca la importante fragmentación en los datos de la asistencia, fundamentalmente en el subsector público, debido a la falta de registros clínicos en red. Esto se debe a que la mayoría de los pacientes concurrían a un centro de salud para el control ambulatorio y a otros centros para la internación donde finalmente ocurrió el fallecimiento.

Se trata de una muestra de población por conveniencia, no alcanzándose a relevar la totalidad de los registros clínicos (124/175 fallecidos durante el año 2014), aunque la muestra alcanzada representa el $71 \%$ del total. Se destaca, además, que en el 15\% de los certificados de defunción a los que tuvimos acceso, existieron problemas de llenado o de codificación de la causa de muerte, aspecto en el que se debe trabajar, ya que se trata de una importante fuente de datos epidemiológicos para el seguimiento y la planificación de las estrategias de eliminación de la epidemia de VIH/Sida en nuestro país.

\section{Conclusiones}

La falta de contacto o contacto irregular con los servicios de salud (no vinculación y no retención) ponen de manifiesto los obstáculos a un acceso continuo y de calidad en la atención de esta población. El análisis de la distribución de los fallecidos en los diferentes pilares de la cascada de cuidados evidencian la importancia estratégica de fortalecer políticas que mejoren la accesibilidad al sistema de salud, promoviendo acciones efectivas de retención en la cadena de cuidados. Algunas características de la población que denotan vulnerabilidad social (situación de calle, uso de drogas, privación de libertad) en una importante proporción de los fallecidos demuestran la necesidad de articular más estrechamente el sistema de salud con la malla de protección social existente en Uruguay.

\section{Agradecimientos}

Dr. Jorge Basso, ministro de Salud. 
Dr. Jorge Quian, subsecretario de Salud.

Andrés Bálsamo, Departamento de Vigilancia en Salud, División Epidemiología - DIGESA, MS.

Adriana Misa, Leticia Rodríguez. Unidad de Información Nacional en Salud, División Epidemiología DIGESA, MS.

Fiorella Cavalleri. Departamento de Métodos Cuantitativos, Facultad de Medicina - UDELAR.

\section{Abstract}

Introduction: early diagnosis of HIV and early and continuos antirretroviral treatment constitute key strategies to avoid dying of AIDS. After a steady increase, HIV-related mortality has remained stable since 2005, although the expected reduction has not occurred. A study was conducted in order to characterize the clinical and epidemiological profile of the population that died of AIDS in Uruguay in 2014 and to assess the distribution of deaths in the continuum of care cascade.

Method: retrospective and observational study, based on information kept in files containing death certificates and medical histories of people who were 18 years old or older and died of AIDS in 2014.

Results: we had access to 124 records of the 175 deaths, finding that 105 corresponded to HIV-related deaths, $77 \%$ of them came from the public subsector and $68 \%$ of them being men. Most of them were young adults (43.7 \pm 11.6 years old) and lived in vulnerable conditions (47.1\% had used drugs; $15.4 \%$ were homeless or in prison; $37.5 \%$ benefited from social plans). In $71.4 \%$ of cases diagnosis was late and $37.1 \%$ died within the first year of diagnosis. $60 \%$ died without having consulted the health system or continued treatment (11.4\% failed to seek health assistance, $48.6 \%$ interrupted treatment).

Conclusions: the increased proportion of patients who died without having sought health assistance or follow up in the health system within a population of social vulnerability evidences the need to adopt comprehensive strategies that have been proved successful to increase access and continuation of treament.

\section{Resumo}

Introdução: o diagnóstico precoce da infecção por VIH e o acesso rápido e continuo ao tratamento antirretroviral são estratégias chaves para evitar a morte por AIDS. Desde 2005, depois de um crescimento continuo, observa-se no Uruguai, a estabilização da mortalidade por AIDS não apresentando o descenso previsto. Este estudo foi realizado com o objetivo de identificar o perfil clínico-epidemiológico da população falecida por AIDS em 2014 no país e avaliar a distribuição das mortes na "cascada do continuo da atenção".
Método: estudo retrospectivo e observacional, analisando a informação dos atestados de óbitos e prontuário de paciente de pessoas com 18 ou mais anos de idade falecidas por AIDS durante 2014.

Resultados: foram identificadas 175 mortes; 124 registros foram analisados, dos quais 105 eram mortes por AIDS, sendo $77 \%$ do subsetor público e $68 \%$ do sexo masculino. Era uma população de adultos jovens (43.7 \pm 11.6 anos) em condições de vulnerabilidade (consumo de drogas eventual $47.1 \%$, morador de rua e privação de liberdade $15.4 \% ; 37.5 \%$ beneficiários de planos sociais). $71.4 \%$ foi diagnosticado tardiamente e $37.1 \%$ faleceu no primeiro ano após o diagnóstico. $60 \%$ faleceu sem haver tido e/ou sem continuidade da atenção de saúde (11.4\% pré vinculação, $48.6 \%$ sem retenção nos cuidados).

Conclusões: a elevada proporção de pacientes falecidos sem ter estabelecido um vínculo e seguimento no sistema de saúde, em uma população com características de vulnerabilidade social, mostra a necessidade de adotar estratégias integrais provadas para melhorar o aceso e a continuidade da atenção.

\section{Bibliografía}

1. Antiretroviral Therapy Cohort Collaboration. Causes of death in HIV-1-infected patients treated with antiretroviral therapy, 1996-2006: collaborative analysis of 13 HIV cohort studies. Clin Infect Dis 2010; 50:1387-96

2. Smith CJ, Ryom L, Weber R, Morlat P, Pradier C, Reiss $\mathbf{P}$, et al. Trends in underlying causes of death in people with HIV from 1999 to 2011 (D:A:D): a multicohort collaboration. Lancet 2014; 384:241-8.

3. Low A, Gavriilidis G, Larke N, B-Lajoie MR, Drouin O, Stover $\mathbf{J}$, et al. Incidence of opportunistic infections and the impact of antiretroviral therapy among HIV-infected adults in low-and middle-income countries: a systematic review and meta-analysis. Clin Infect Dis 2016; 62(12):1595-603.

4. Roger AJ, Lodwick R, Schechter M, Deeks S, Amin J, Gilson R, et al. Mortality in well controlled HIV in the continuous antiretroviral therapy arms of the SMART and ESPRIT trials compared with the general populations. AIDS 2013; 27(6):973-9.

5. Palella FJ Jr, Delaney KM, Moorman AC, Loveless MO, Fuhrer J, Satten GA, et al. Declining morbidity and mortality among patients with advanced human inmunodeficiency virus infection. HIV Outpatient Study Investigators. N Engl J Med 1989; 338(13):853-60.

6. Montaner JS, Lima VD, Harrigan PR, Lourenço L, Yip B, Nosyk B, et al. Expansion of HAART coverage is associated with sustained decreases in HIV/AIDS morbidity, mortality and HIV transmission: the "HIV Treatment as Prevention" experience in a Canadian setting. PLoS One 2014; 9(2):e87872. 
7. Palella FJ Jr, Baker RK, Moorman AC, Chmiel JS, Wood KC, Brooks JT, et al. Mortality in the highly active antiretroviral therapy era: changing causes of death and disease in the HIV outpatient study. J Acquir Immune Defic Syndr 2006; 43:27-34.

8. Chadborn TR, Delpech VC, Sabin CA, Sinka K, Evans BG. The late diagnosis and consequent short-term mortality of HIV-infected heterosexuals (England and Wales, 2000-2004). AIDS 2006; 20:2371-9.

9. Ulett KB, Willig JH, Lin HY, Routman JS, Abroms S, Allison J, et al. The therapeutic implications of timely linkage and early retention to HIV care. AIDS Patient Care STDS 2009; 23:41-9.

10. Torian LV, Wiewel EW, Liu KL, Sackoff JE, Frieden TR. Risk factors for delayed initiation of medical care after diagnosis of human immunodeficiency virus. Arch Intern Med 2008; 168:1181-7.

11. Mugavero MJ, Davila JA, Nevin CR, Giordano TP. From access to engagement: measuring retention in outpatient HIV clinical care. AIDS Patient Care STDS 2010; 24:607-13.

12. Durán A, Pérez E, Adaszko A. Mortalidad por sida en la Argentina. Perfil de las personas fallecidas por sida en el Área Metropolitana de Buenos Aires durante el año 2010. Bs. As: Ministerio de Salud, 2014. Disponible en: http://www.msal.gob.ar/sida/images/stories/4-publicaciones/pdf/2014-11-13_estudio-mortalidad-sida.pdf [Consulta: abril 2018].

13. Gardner EM, McLees MP, Steiner JF, Del Rio C, Burman WJ. The spectrum of engagement in HIV care and its relevance to test-and-treat strategies for prevention of HIV infection. Clin Infect Dis 2011; 52:793-800.

14. Mugavero MJ, Amico KR, Horn T, Thompson MA. The state of engagement in HIV care in the United States: from cascade to continuum to control. Clin Infect Dis 2013; 57:1164-71.

15. Egger M, May M, Chêne G, Phillips AN, Ledergerber B, Dabis F, et al. Prognosis of HIV-1-infected patients starting highly active antiretroviral therapy: a collaborative analysis of prospective studies. Lancet 2002; 360:119-29.

16. Kitahata MM, Gange SJ, Abraham AG, Merriman B, Saag MS, Justice AC, et al. Effect of early versus deferred antiretroviral therapy for HIV on survival. N Engl J Med 2009; 360:1815-26.

17. HIV-CAUSAL Collaboration, Ray M, Logan R, Sterne JA, Hernández-Díaz S, Robins JM, et al. The effect of combined antiretroviral therapy on the overall mortality of HIV-infected individuals. AIDS 2010; 24:123-37.

18. Krentz HB, MacDonald J, John Gill M. High mortality among human immunodeficiency virus (HIV)-infected individuals before accessing or linking to HIV care: a missing outcome in the cascade of care? Open Forum Infect Dis 2014; 1(1):ofu011.

19. Ministerio de Salud Pública. Departamento de Vigilancia en Salud-Programa ITS-VIH/SIDA. Situación epidemiológica del VIH/SIDA en Uruguay. Bol Epidemiol 2015; 1-44.

20. Uruguay. Ministerio de Salud Pública. Estudio de Carga Global de Enfermedad. Programa de Prevención de Enfermedades No Transmisibles. Montevideo: MSP, 2010. Disponible en: http://www.msp.gub.uy/sites/default/files/archivos_adjuntos/Estudio_de_Carga_Global.pdf [Consulta: abril 2018].

21. Cesar C, Koethe JR, Giganti MJ, Rebeiro P, Althoff KN, Navapravnik S, et al. Health outcomes among HIV-positive Latinos initiating antiretroviral therapy in North America versus Central and South America. J Int AIDS Soc 2016; 19(1):20684.

22. Granich RM, Gilks CF, Dye C, De Cock KM, Williams BG. Universal voluntary HIV testing with immediate antiretroviral therapy as a strategy for elimination of HIV transmission: a mathematical model. Lancet 2009; 373:48-57.

23. McNairy ML, Lamb MR, Gachuhi AB, Nuwagaba-Biribonwoha $\mathrm{H}$, Burke S, Mazibuko S, et al. Effectiveness of a combination strategy for linkage and retention in adult HIV care in Swaziland: the Link4Health cluster randomized trial. PLoS Med 2017; 14(11):e1002420.

24. Giordano TP. Strategies for linkage to and engagement with care: focus on intervention. Top Antivir Med 2018; 26(2):62-5.

25. Kredo T, Ford N, Adeniyi FB, Garner P. Decentralising HIV treatment in lower-and middle- income countries. Cochrane Database Syst Rev 2013; (6):CD009987. doi: 10.1002/14651858.CD009987.pub2.

\section{Contribución de autores}

Todos los autores participaron en igual medida en las etapas de concepción, diseño, ejecución, análisis, redacción y revisión.

Daniel Pérez, https://orcid.org/0000-0003-3371-6614

Juan Meré, https://orcid.org/0000-0001-8719-5928

Victoria Frantchez, https://orcid.org/0000-0001-9040-4524

Elisa Cabeza, https://orcid.org/0000-0002-5256-0056

Carolina Iglesias, https://orcid.org/0000-0002-2498-4046

Susana Cabrera, https://orcid.org/0000-0002-2126-0640 
Anexo. Definiciones operativas.

\begin{tabular}{|c|c|}
\hline Personas con diagnóstico de VIH: & $\begin{array}{l}\text { Constancia de estudio confirmatorio realizado en el Departamento de Laboratorios de Salud Pública o persona con } \\
\text { prueba de screening de VIH reactiva y enfermedad marcadora de Sida y/o signos de inmunodepresión (signos B de } \\
\text { la estadificación de los Centers for Diseases Control - CDC) y/o recuento de CD } 4 \leq 200 \text { células/ml. }\end{array}$ \\
\hline Sida: & $\begin{array}{l}\text { Síndrome de inmunodeficiencia adquirida definido por las enfermedades marcadoras (signos } C \text { de la estadificación } \\
\text { de los Centers for Diseases Control - CDC) y/o recuento de CD4 } \leq 200 \text { células } / \mathrm{ml} \text {. }\end{array}$ \\
\hline Diagnóstico tardío: & $\begin{array}{l}\text { Recuento de CD } 4 \leq 200 \mathrm{cel} / \mathrm{ml} \text { en los } 12 \text { primeros meses posteriores al diagnóstico, } \\
\text { y/o? } \\
\text { enfermedad oportunista en los } 12 \text { primeros?meses posteriores al diagnóstico, ? } \\
\text { y/o } \\
\text { fallecidos por Sida con diagnóstico de VIH en los } 12 \text { meses previos a la ?defunción. }\end{array}$ \\
\hline $\begin{array}{l}\text { Personas vinculadas al sistema de } \\
\text { atención: }\end{array}$ & $\begin{array}{l}\text { Tuvieron al menos un recuento de CD4 o una determinación de carga viral o una consulta por VIH en los } 12 \text { meses } \\
\text { siguientes al diagnóstico. }\end{array}$ \\
\hline $\begin{array}{l}\text { Personas retenidas en el sistema } \\
\text { de atención: }\end{array}$ & $\begin{array}{l}\text { Dos o más consultas por VIH anuales, } \\
\text { y/o } \\
\text { dos o más recuentos de CD } 4 \text { y carga viral anualmente, } \\
\text { y/o } \\
\text { han recogido el TARV } 4 \text { o mas veces anualmente. }\end{array}$ \\
\hline Adherencia al tratamiento: & $\begin{array}{l}\text { Constancia en la toma de la TARV, considerando buena adherencia al registro en la historia clínica, sin } \\
\text { interrupciones por causas vinculadas al paciente, } \leq 1 \text { falta a control en cada año de TARV. }\end{array}$ \\
\hline Muerte pre vinculación: & Sin una consulta o un recuento de CD4 o carga viral antes de la muerte. \\
\hline Muerte no retenido: & $\begin{array}{l}\text { Tuvo vinculación, pero no permanencia en el sistema. El paciente pudo haber entrado y salido del seguimiento y } \\
\text { pudo haber tenido TARV. Excluye pacientes bajo cuidados en los últimos } 2 \text { años. }\end{array}$ \\
\hline $\begin{array}{l}\text { Carga viral de VIH indetectable } 0 \\
\text { suprimida: }\end{array}$ & Carga viral menor a 20 copias $/ \mathrm{ml}$. \\
\hline
\end{tabular}

